\title{
Intrinsic noise of a superheated droplet detector for neutron background measurements in massively shielded facilities
}

\author{
Ana C. Fernandes ${ }^{1, *}$, Tomoko A. Morlat ${ }^{1}$, Miguel Felizardo ${ }^{1}$, Andreas $\mathrm{Kling}^{1}$, José G. Marques ${ }^{1}$, Maria I. Prudêncio ${ }^{1}$, Rosa \\ Marques $^{1}$, Fernando P. Carvalho ${ }^{1}$, Ignácio Lázaro Roche ${ }^{2}$ and Thomas A. Girard ${ }^{3}$ \\ ${ }^{1}$ Centro de Ciências e Tecnologias Nucleares, Instituto Superior Técnico, Universidade de Lisboa, 2695-066 Bobadela, Portugal \\ ${ }^{2}$ Laboratoire Souterrain à Bas Bruit, University of Nice, University of Avignon, Centre National de la Reserche Scientifique, Aix- \\ Marseille University, Observatoire de la Côte d'Azur, 84400 Rustrel, France \\ ${ }^{3}$ Departamento de Física, Faculdade de Ciências, Universidade de Lisboa, 1749-016 Lisboa, Portugal
}

\begin{abstract}
Superheated droplet detectors are a promising technique to the measurement of low-intensity neutron fields, as detectors can be rendered insensitive to minimum ionizing radiations. We report on the intrinsic neutron-induced signal of $\mathrm{C}_{2} \mathrm{ClF}_{5}$ devices fabricated by our group that originate from neutron- and alpha-emitting impurities in the detector constituents. The neutron background was calculated via Monte Carlo simulations using the MCNPX-PoliMi code in order to extract the recoil distributions following neutron interaction with the atoms of the superheated liquid. Various nuclear techniques were employed to characterise the detector materials with respect to source isotopes $\left({ }^{238} \mathrm{U},{ }^{232} \mathrm{Th}\right.$ and $\left.{ }^{147} \mathrm{Sm}\right)$ for the normalisation of the simulations and also light elements (B, Li) having high $(\alpha, n)$ neutron production yields. We derived a background signal of $\sim 10^{-3} \mathrm{cts} /$ day in a 1 liter detector of $1-3 \mathrm{wt} . \% \mathrm{C}_{2} \mathrm{ClF}_{5}$, corresponding to a detection limit in the order of $10^{-8} \mathrm{n} \mathrm{cm}^{-2} \mathrm{~s}^{-1}$. Direct measurements in a massively shielded underground facility for dark matter search have confirmed this result. With the borosilicate detector containers found to be the dominant background source in current detectors, possibilities for further noise reduction by $\sim 2$ orders of magnitude based on selected container materials are discussed.
\end{abstract}

\section{Introduction}

A superheated droplet detector (SDD) consists of a homogeneous suspension of micrometric superheated liquid droplets inside a viscous gel of matched density, each droplet functioning as a small bubble chamber [1, 2]. These detectors are traditionally used for personal neutron dosimetry $[3,4]$, but have also been employed in high energy neutron spectrometry $[5,6]$, registration of high and intermediate heavy ions [7] and direct search for dark matter $[8,9]$. At $\mathrm{C}^{2} \mathrm{TN}$ superheated droplet detectors (SDD) of 1-3 wt. $\% \mathrm{C}_{2} \mathrm{ClF}_{5}$ in a food gel-based matrix are developed and operated with an acoustic instrumentation for signal detection. The detectors have been used for the detection of rare events in the context of astrophysics [9] and more recently for alpha particle $(\alpha)$ measurements [10].

The response of the device is based on the Seitz "thermal spike" model $[3,11]$ : if the energy deposition is sufficiently large within a sufficiently short distance in the droplet, the droplet vaporises. These limits depend on the liquid and its thermodynamic degree of superheating: typically the higher the superheat, the lower the energy required for bubble nucleation. A selection of the thermodynamic operation conditions can render the SDD insensitive to energetic muons, electrons and photons $<6$ $\mathrm{MeV}$ that plague most rare event detection experiments targeting heavy ionising radiations. We therefore obtain a low-noise instrument for the characterisation of neutron environments at extremely low intensities where the reduced radiation intensity hampers the use of most experimental methods. The application is particularly interesting for shielded facilities employed in low-noise gamma-spectroscopy [12], intrinsic soft error rate measurements in electronic devices [13], investigation of neutrino and neutrino-less physical processes and direct search for dark matter [14]. The intrinsic detector background becomes critical, for it determines the ultimate detection limit thereby affecting the measurement accuracy and precision.

The SDD signal was measured at an underground facility in LSBB [15] following various efforts to reduce the environmental neutron radiation that originated from the cavern structural materials $[16,17]$ and the $\alpha$ background induced by atmospheric radon that might diffuse into the detector gel [18]. The neutron signal corresponding to the experimental set-up was calculated independently, using the stochastic MCNP-5 code [19] to model neutron transport from and within the entire facility [20]. The calculations showed that $>85 \%$ of the SDD signal originated from its own materials, namely the borosilicate glass container. It was therefore concluded that the measurement at this massively

Corresponding author: anafer@,ctn.tecnico.ulisboa.pt 
shielded facility provided a direct determination of the detector intrinsic noise.

For the simulations, additional codes were employed to extract the reaction rates from the neutron spectra. These included FLXPRO [21] to manage reaction cross sections and uncertainties, and SPECTER (based on the ENDF-5 cross section data from the 1980's) to derive the distribution of nuclear recoils that ultimately produce the detector signal [22]. Neutron production yields from $(\alpha, n)$ reactions were obtained from the USD code [23] which was the only publicly-available software providing $(\alpha, n)$ spectra for $\alpha$ energies covering the ${ }^{238} U$ and ${ }^{232} \mathrm{Th}$ decay chains. The total neutron yields from USD have the same order of magnitude (discrepancy of $25-45 \%)$ as those from other $(\alpha, n)$ codes and experimental data; however, unrealistic sharp peaks are observed at the lower end of the energy distribution. Although these features spread out in the hydrogenous gel of the SDD [20] they are expected to affect the characterisation of the bulk superheated liquid devices under development [24].

In this paper we investigate the intrinsic background of the SDD using the MCNPX-PoliMi-2.0 code [25] to extract directly the recoil distribution using the most recent cross section and angular data. The $(\alpha, n)$ data is mostly obtained from the SOURCES-4A code extended to $10 \mathrm{MeV} \alpha$ 's [26]. New experimental data regarding emitters and relevant $(\alpha, n)$ isotopes in the gel is now included in the model. The revised detection limit is determined for the current detector design, and further developments towards improving its performance are identified.

\section{The SDD}

\subsection{General description}

As described in Section 1, the threshold conditions required for the phase transition of a droplet set thermodynamically-defined minimum values for the radiation energy and linear energy transfer. Our SDDs are usually operated at $9{ }^{\circ} \mathrm{C}$ and 2 bar. Pressurization improves the detector life and reduces $\mathrm{Rn}$ diffusion into the gel. The SDD sensitivity to radiations simultaneously depositing less than $8 \mathrm{keV}$ at less than $160 \mathrm{keV} \mathrm{\mu m}^{-1}$ is inhibited, rendering the detector sensitive only to nuclei recoiling after neutron interactions, and $\alpha$ 's. In the case of neutrons, the threshold conditions define a critical recoil energy $\left(E_{\text {rec }}\right)$ hence a minimum neutron energy $\left(E_{\min }\right)$ for the induction of a nucleation - the relation between $E_{\text {rec }}$ and $E_{\text {min }}$ being determined by the kinematics of the nuclear interaction [27].

In order to gain sensitivity the concentration of superheated liquid is larger than that of commercial devices $(<0.1$ wt. $\%)$. The gel becomes opaque inhibiting the visual detection of the events; a low-frequency acoustic instrumentation is employed to detect the shock wave formed by the bubble expansion in the gel [28].

Figure 1 shows a $150 \mathrm{ml} \mathrm{SDD}$ with 2 wt. $\% \mathrm{C}_{2} \mathrm{ClF}_{5}$. The container is made of laboratory-grade borosilicate glass (Schott Duran, Germany). The cap is modified with a feedthrough that allows detector pressurization, pressure monitoring and signal acquisition with a microphone embedded in a $1 \mathrm{~cm}$ glycerine layer that covers the gel.

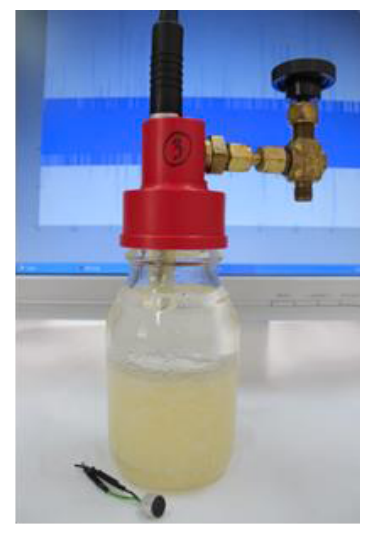

Fig. 1. A Sm-doped $150 \mathrm{ml}$ SDD equipped with microphone and valve for pressurisation. The raw acoustic signal is displayed in the background. A discard microphone is shown in front of the detector.

The detector lifetime is limited by the fracture of the gel which in turn is promoted by the growth of gas bubbles in an exposed detector. Underground testing of an SDD with improved fabrication protocols towards lifetime extension [29] yielded an intrinsic lifetime of 100 days.

Signal treatment and analysis allowed to determine the characteristic frequency signature of events originated by radiation and identify them among other acoustic, seismic, or gel-related noises [30]. In addition, recoil and $\alpha$-induced events can be discriminated based on signal amplitudes, with $\alpha$ 's producing larger amplitudes as a result of the production of various protobubbles along the Bragg peak [31, 32].

Further details on SDD fabrication, instrumentation and particle discrimination can be found in Ref. 9 and references therein. A modification was recently introduced relatively to the standard fabrication protocol, with the droplet shearing step occurring at a higher temperature $\left(60^{\circ} \mathrm{C}\right)$ than standard $\left(45^{\circ} \mathrm{C}\right)$ in order to decrease the gel stiffness.

The particle discrimination capability (due to the existence of a gap between recoil and $\alpha$-amplitude distributions) is linked to the droplet size distribution [33]. The size distribution was recently measured by optical microscopy for $150 \mathrm{ml}$ devices (Fig. 2); a gaussian fit yields an average radius of $4.6 \mu \mathrm{m}$. The average droplet size is significantly smaller than previously reported for 1 liter standard devices $(30 \mu \mathrm{m})$; a possible cause for the discrepancy is the increased efficiency of the droplet shearing due to the smaller device volume and gel stiffness. 


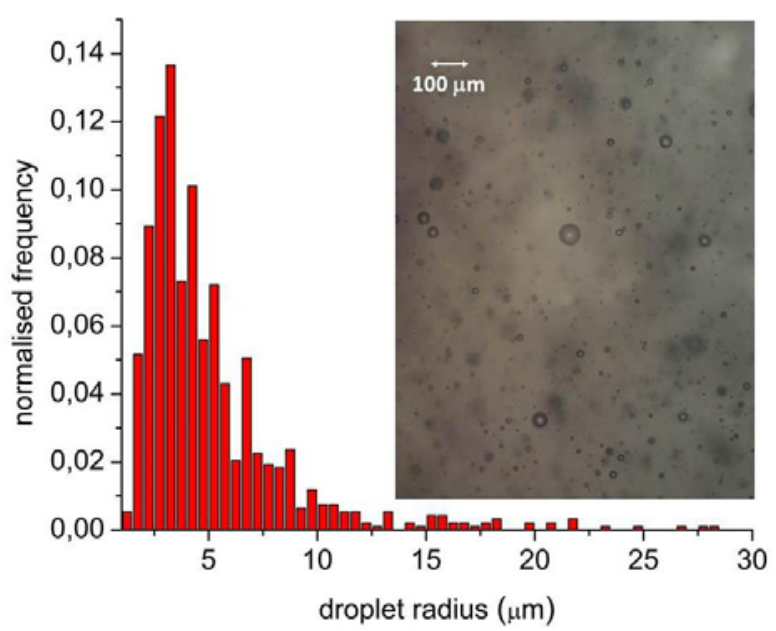

Fig. 2. Droplets (magnification 100x) and droplet size distribution in a $150 \mathrm{ml}$ fresh SDD (sample size: 930 droplets).

The size distribution is found to be preserved after a recompression stage that reliquifies the gas bubbles allowing the regeneration of an exposed detector. This facilitates neutron spectrometry, in which the temperature-varying method at fixed pressure is employed to modify $\mathrm{E}_{\min }$. As the temperature increases, neutrons of lower energies are gradually included in the measurement (Fig. 3). From the difference between the detector signals at different temperatures the fluence rate of neutrons between the corresponding $E_{\min }$ can be derived [34]. However, as measurements at moderate temperatures are expected to decrease the SDD lifetime, the detector regeneration possibility is an interesting alternative to the application of fresh devices with potentially different sensitivities.

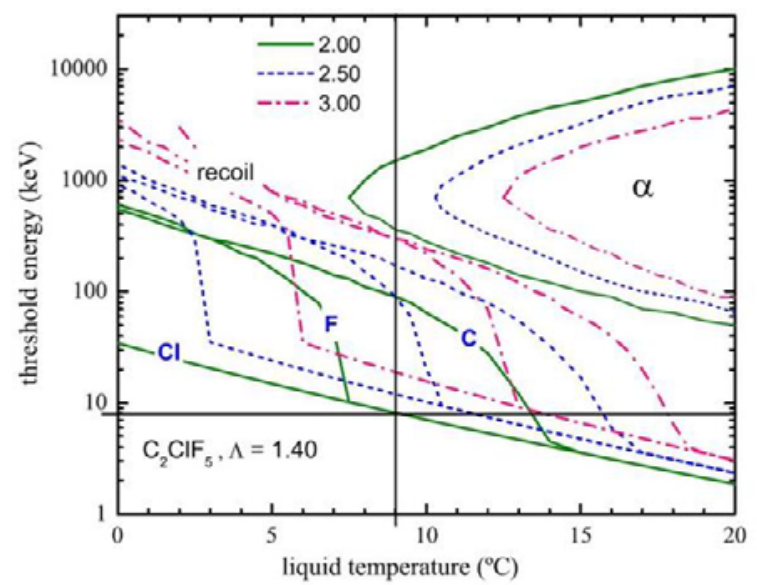

Fig. 3. Threshold energy of nuclear recoils and alpha particles for $\mathrm{C}_{2} \mathrm{ClF}_{5}$ as a function of temperature, at different pressures. The vertical and horizontal lines denote the standard operating conditions $\left(9^{\circ} \mathrm{C}\right.$ and 2 bar).

\subsection{Materials}

The choice and characterisation of materials is crucial for the development of a low-noise neutron detector, as most materials contain traces of radioisotopes that produce neutrons either by spontaneous fission or via $(\alpha, n)$ reactions. We are mostly concerned with ${ }^{238} \mathrm{U}$ and ${ }^{232} \mathrm{Th}$ due to the emission of $\alpha$ 's in the course of the decay chain and neutron emission following the spontaneous fission of ${ }^{238} \mathrm{U}$. Samarium is a natural emitter of low energy $\alpha$ 's (from ${ }^{147} \mathrm{Sm}$, with a natural abundance of $14.6 \%$ ) whose presence in the gel justifies evaluation [23]. The presence of light elements with high $(\alpha, n)$ production yields - namely Boron - in material compositions was also considered.

Neutron activation analysis, gamma- and $\alpha$ spectrometry and ion-coupled plasma spectrometry (ICP-MS) were used to quantify the emitters, whereas the determination of $\mathrm{B}$ and $\mathrm{Li}$ employed ion beam analysis techniques and ICP-MS. The results are summarised in Table 1. When concentrations are below the detection limits, the latter are quoted. Materials' composition and density were extracted from Ref. 20; that from the gel was modified to include the superheated liquid as required for MCNPX-PoliMi. For neutron transport and recoil calculation, the revised composition (in weight) for an SDD with 2 wt.\% superheated liquid is: $8.92 \% \mathrm{H}, 33.43 \% \mathrm{C}, 0.74 \% \mathrm{~N}$, $55.22 \mathrm{O}, 1.23 \% \mathrm{~F}$ and $0.46 \% \mathrm{Cl}$. The possible traces of $\mathrm{B}$ and $\mathrm{Li}$ in the gel were considered only to derive the $(\alpha, n)$ source term (Section 3.2.1).

Table 1. Measured concentrations (in $\mu \mathrm{g} / \mathrm{g}$ ) of neutron and $\alpha$ emitters and selected light elements in the detector materials.

\begin{tabular}{|c|c|c|c|}
\hline $\begin{array}{c}\text { Isotope or } \\
\text { Element }\end{array}$ & Borosilicate & Gel & Microphone \\
\hline${ }^{238} \mathrm{U}$ & $0.220 \pm 0.033$ & $<0.001$ & $<1.09$ \\
\hline${ }^{232} \mathrm{Th}$ & $0.310 \pm 0.027$ & $<0.01$ & - \\
\hline${ }^{147} \mathrm{Sm}$ & - & $<0.001$ & - \\
\hline $\mathrm{B}$ & $\begin{array}{c}(4.1 \pm 0.2) \\
\mathrm{x} 10^{4}\end{array}$ & $<0.03$ & $\sim 0$ \\
\hline $\mathrm{Li}$ & - & $<0.005$ & - \\
\hline
\end{tabular}

\section{Intrinsic neutron-induced noise}

\subsection{Measurement}

\subsubsection{Brief description of the experimental set-up}

The SDD background noise was measured in the GESA room of LSBB, equipped with a water-based neutron shield. The facility is located at $500 \mathrm{~m}$ depth in calcite rock, corresponding to $1500 \mathrm{~m}$ water-equivalent (mwe). A schematic view of GESA with the experimental set-up is shown in Fig. 4. A detailed description of the facility including dimensions can be found in Refs. 9, 20. The cavern walls and ceiling are finished with concrete and a steel lining. Fifteen SDDs of 1 liter, fabricated in an adjacent clean room, sat on a 
thermostatic water bath of 750 liter for temperature control, with the water level covering them up to the glycerine level. Each detector glass container weighted $740 \mathrm{~g}$.

A water shield was installed around the tank for protection against environmental neutrons from the cavern structural materials (mostly from the concrete). The temperature of the water bath and individual detectors pressure were monitored in order to evaluate the stability of the thermodynamic operation conditions. This information, together with the signal from each detector was acquired continuously and available on-line and remotely.

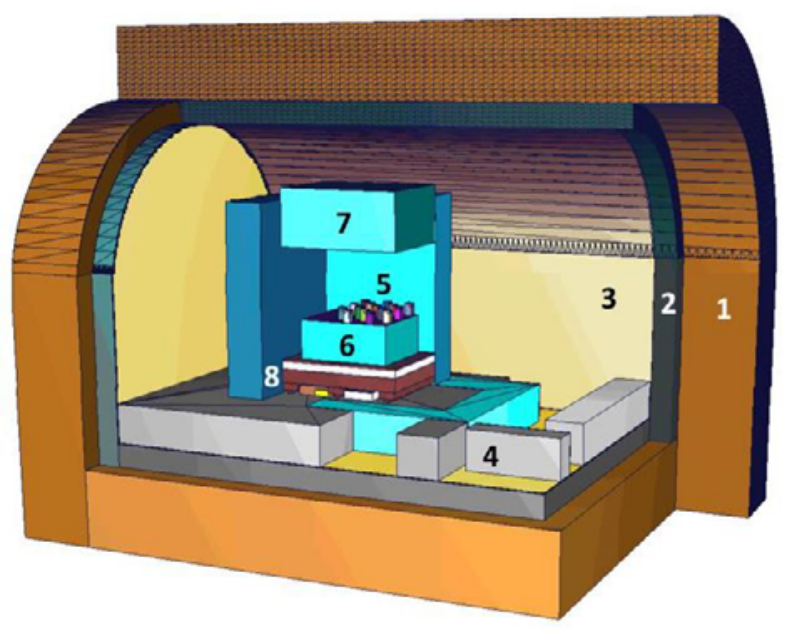

Fig. 4. Schematic view of the GESA room with fifteen SDDs in a water bath surrounded by a neutron shield (some parts were removed from the figure for clarity). 1: rock; 2: concrete; 3: steel lining; 4: concrete floor structures; 5 : detectors; 6 : tank (water below the detectors); 7: water shield: 8: wooden tank support with various polyethylene and paraffin layer/inserts.

\subsubsection{Radiation backgrounds}

The water-based shielding, with a thickness of 50-75 $\mathrm{cm}$ was designed using a detailed MCNP model of the facility (also displayed in Fig. 4). MCNP outputs were normalised to the measured ${ }^{238} \mathrm{U}$ and ${ }^{232} \mathrm{Th}$ concentrations in the various structural, shield and detector materials. A reduction of the neutron field in the facility by 2 orders of magnitude was achieved, with ondetector neutron fluence rate values in the order of $10^{-8} \mathrm{~cm}^{-2} \mathrm{~s}^{-1}$. The overall calculated event rate is $0.372 \pm 0.097 \mathrm{evt} / \mathrm{kgd}$, of which $86 \%$ originate in the detector glass [20].

Efforts to reduce the $\alpha$-induced signal due to the diffusion of atmospheric radon into the detector walls include purging the air 10 times per day (reducing the maximum $\mathrm{Rn}$ concentration from 1000 to $60 \mathrm{~Bq} \mathrm{~m}^{-3}$ ) and circulating the water bath in order to replace the top 1 cm layer each minute [9, 18, 31].

The influence of Rn adsorption on the glass container stemming from the water bath and consequent implantation of the decay daughters originating $(\alpha, n)$ neutrons is evaluated for the first time. For equilibrium concentrations of $\mathrm{Rn}$ in air and water [35], the amount of
$\mathrm{Rn}$ available for adsorption is $22 \mathrm{mBq} / \mathrm{liter}$. This is actually an upper limit, since water circulation is neglected. The total Rn activity in the pool water is $\sim 16$ Bq. Due to the geometrical arrangement the detectors occupy only about $15 \%$ of the pool volume reducing further the amount of $\mathrm{Rn}$ available for adsorption at the glass to $3 \mathrm{~Bq}$ (for the remaining volume adsorption at the pool walls - which have a surface area ca. five times larger than the detectors - exhibits an alternative sink). In addition, the amount of $\mathrm{Rn}$ descendants implanting in the glass is less than $50 \%$ of the adsorbed. Comparing the $<2 \mathrm{~Bq}$ of implanted activity from $\mathrm{Rn}$ in the surrounding water to the $44.5 \mathrm{~Bq} \mathrm{Rn}$ contained in the $11.1 \mathrm{~kg}$ of glass in equilibrium $\left(2.74 \mathrm{~Bq} \mathrm{~kg}^{-1}\right.$ from ${ }^{238} \mathrm{U}$ and $1.27 \mathrm{~Bq} \mathrm{~kg}^{-1}$ from ${ }^{232} \mathrm{Th}$ decay chains) shows that any additional $\mathrm{Rn}$ activity due to an eventual adsorption of $\mathrm{Rn}$ and its subsequent recoil implantation at the water/glass interface has a negligible influence. Finally, the SDDs are fabricated in the clean room with similar $\mathrm{Rn}$ levels for various days before installation in the experiment, thereby "losing memory" of previous Rn exposure (half-life of ${ }^{222} \mathrm{Rn}$ from ${ }^{238} \mathrm{U}$ is $3.8 \mathrm{~d}$; of ${ }^{220} \mathrm{Rn}$ from ${ }^{232} \mathrm{Th}$ is $56 \mathrm{~s}$ ).

Cosmic muons interacting with rock originate high energy neutrons that can contribute to the detector signal. Estimates of the corresponding neutron field were based on data from Refs. 36, 37, and are reported in detail in Ref. 20. Data from other underground facilities were interpolated in order to derive the neutron energy distribution up to $3 \mathrm{GeV}$ for GESA's rock properties (namely density: $2.61 \mathrm{~g} \mathrm{~cm}^{-3}$ and average atomic number: 23.4). MCNPX [38] was employed to estimate the neutron-induced signal in the SDDs, yielding an event rate that is $0.6 \%$ of total.

\subsubsection{Experimental results}

A measurement stage of 75 days was performed using fifteen SDDs of 1 liter comprising a total of $215 \mathrm{~g}$ superheated liquid. Three detectors failed within days as a result of overpressure and were excluded from the measurement data resulting in a liquid loss of $45 \mathrm{~g}$. Another detector of $11.5 \mathrm{~g}$ liquid failed after 48 days of measurements; its results have been accounted for. There is a trend for the increase of detector pressure with time as bubbles are formed and the gel swells. Data at $>2.2$ bar has been excluded in order to maintain the recoil energy threshold of $8 \mathrm{keV}$. This yield an exposure loss down to $6.71 \mathrm{kgd}$.

A total of 1982 events were registered. Of these, 1171 are correlated among the various SDDs indicating an origin diverse from radiation. Signal analysis indicated that $95 \%$ of the uncorrelated events corresponded to environmental noise events, gel fractures and gas microleaks. The characteristic frequency signature allowed to identify 30 nucleations, of which 29 were induced by $\alpha$ 's. An overall result of 1 recoil event is derived corresponding to $0.15 \mathrm{evt} / \mathrm{kgd}$ that originate from the detector container, as described in Section 3.1.2. 


\subsection{Calculation}

\subsubsection{Neutron production yields}

The intrinsic neutron-induced noise of a 1 liter SDD at $2 \mathrm{wt. \%}$ liquid originating from its container, gel and microphone was calculated using MCNPX-PoliMi. A simple geometry model was used: the SDD glass container is represented as a box of square cross section ( $8 \mathrm{~cm}$ internal size) of $5 \mathrm{~mm}$ thickness having gel up to $12 \mathrm{~cm}$ height and a $2 \mathrm{~cm}$ glycerine layer embedding a punctual microphone at the geometric centre.

The neutron source description included the Watt spontaneous fission spectrum with tabulated parameters (the intensity being derived from the measured ${ }^{238} \mathrm{U}$ concentrations and decay data), as well as $(\alpha, n)$ yields and spectra calculated using the modified SOURCES-4A code. Isotropic emission was considered. Secular equilibrium in the decay chains was assumed. This is the case for ${ }^{238} \mathrm{U}$ in the borosilicate glass used, for which there is no evidence of disequilibrium in the ${ }^{226} \mathrm{Ra}$ subchain [39]. With respect to the microphone material, SOURCES lacks neutron production data in $\mathrm{Fe}$ and the USD code was used instead.

Production yields and energy distributions are shown in Table 2 and Fig. 5. For the gel, upper limits were derived for the detection thresholds of emitters and light elements (Table 1). The latter were found to have no impact on the calculated neutron yields.

The complex structure of the USD data for $\mathrm{Fe}$ contrasts with the smooth distribution retrieved by SOURCES for Th (and in general); a possible cause for this effect could be a deficient calculation of the continuous $\alpha$ energy loss in the material by the USD code [40]. The Sm-induced distribution is a particular case where the sharp features retrieved by SOURCES are due to the fact that at low $\alpha$ energies only a very limited number of target isotopes and states contributes to the production of neutrons and extends up to low energies. The maxima shown in Fig. 5 represent (by increasing energy) the contribution of the first excited and ground states of ${ }^{20 / 21} \mathrm{Ne}$ isotopes and of ${ }^{16} \mathrm{O}[40,41]$.

Table 2. Neutron yields $\left(n \mu g^{-1} y^{-1}\right)$ from spontaneous fission and decay-induced $(\alpha, n)$ reactions due to the presence of emitters in the SDD materials. Secular equilibrium is assumed for ${ }^{238} \mathrm{U}$ and ${ }^{232} \mathrm{Th}$ decay chains.

\begin{tabular}{|c|c|c|c|}
\hline $\begin{array}{c}\text { Reaction / } \\
\text { Material }\end{array}$ & ${ }^{{ }^{238}} \mathbf{U}$ & ${ }^{{ }^{232}} \mathbf{T h}$ & ${ }^{{ }^{\text {nat }} \mathbf{S m}}$ \\
\hline $\begin{array}{c}\text { Spontaneous } \\
\text { fission }\end{array}$ & 0.430 & $3.84 \times 10^{-6}$ & 0 \\
\hline $\begin{array}{c}(\alpha, \mathrm{n}) \text { in } \\
\text { borosilicate }\end{array}$ & 3.185 & 1.142 & $4.86 \times 10^{-5}$ \\
\hline $\begin{array}{c}(\alpha, \mathrm{n}) \text { in gel } \\
\begin{array}{c}(\alpha, \mathrm{n}) \text { in } \\
\text { microphone }\end{array}\end{array}$ & 0.633 & 0.267 & $2.98 \times 10^{-5}$ \\
\hline
\end{tabular}

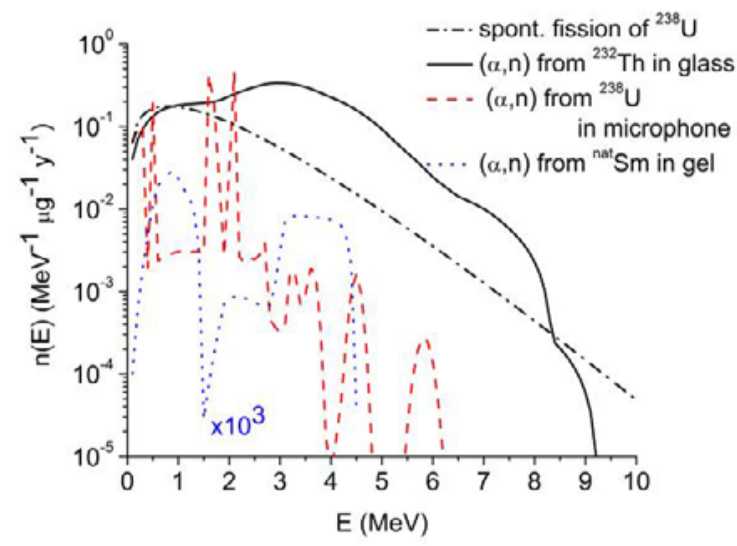

Fig. 5. Energy distribution of $(\alpha, n)$ neutrons due to the presence of ${ }^{238} \mathrm{U}$ and ${ }^{232} \mathrm{Th}$ (in secular equilibrium) and ${ }^{\text {nat }} \mathrm{Sm}$ for various SDD materials. The Watt distribution representing the spontaneous fission of ${ }^{238} \mathrm{U}$ is also represented.

\subsubsection{Event-producing reactions}

The various neutron interactions with the liquid atoms were evaluated with respect to the threshold conditions referred in Section 2.1. We considered elastic and inelastic scattering in $\mathrm{F}, \mathrm{Cl}$ and $\mathrm{C}$, and the transmutation reactions with positive Q-value: $(n, \alpha)$ and $(\mathrm{n}, \mathrm{p})$ in ${ }^{35} \mathrm{Cl}$. Previous investigations [20] allow to reduce the number of the relevant contributing reactions; their $E_{\text {rec }}$ and corresponding $E_{\min }$ are shown in Table $3 . E_{\min }$ is in the order of $10-100 \mathrm{keV}$ for scattering and 0 for the transmutation reactions.

Table 3. Threshold recoil and minimum neutron energy for the event-producing reactions in $\mathrm{C}_{2} \mathrm{ClF}_{5}$ at $9^{\circ} \mathrm{C}$ and 2 bar (elastic scattering: $(n, n)$; inelastic scattering: $\left.\left(n, n^{\prime}\right)\right)$.

\begin{tabular}{|c|c|c|}
\hline Reaction & $\mathbf{E}_{\text {rec }}(\mathbf{k e V})$ & $\mathbf{E}_{\mathbf{m i n}}(\mathbf{k e V})$ \\
\hline${ }^{\mathrm{nat}} \mathrm{C}(\mathrm{n}, \mathrm{n})^{\mathrm{nat}} \mathrm{C}$ & 115 & 402 \\
\hline${ }^{\mathrm{nat}} \mathrm{F}(\mathrm{n}, \mathrm{n})^{\mathrm{nat}} \mathrm{F}$ & 8 & 43 \\
\hline${ }^{\mathrm{nat}} \mathrm{Cl}(\mathrm{n}, \mathrm{n})^{\mathrm{nat}} \mathrm{Cl}$ & 8 & 75 \\
\hline${ }^{\mathrm{nat}} \mathrm{F}\left(\mathrm{n}, \mathrm{n}^{\prime}\right)^{\mathrm{nat}} \mathrm{F}$ & 8 & 120 \\
\hline
\end{tabular}

\subsubsection{Neutron fluence and event rates}

The detection efficiency curve of the SDD deviates from a perfect step function due to statistical fluctuations in the energy deposition process. Experimental results yield a best fit to the function 1-exp[- $\left.\Gamma\left[\mathrm{E} / \mathrm{E}_{\mathrm{rec}}-1\right)\right]$ for $\mathrm{E} \geq \mathrm{E}_{\mathrm{rec}}$ with $\Gamma=4.2 \pm 0.3$, and 0 otherwise [9]. The calculated recoil distribution was convoluted with the efficiency curve when calculating the detector signal.

The on-detector calculated neutron energy distribution calculated with MCNPX is shown in Fig. 6 in the lethargy representation. It has the characteristic shape of a moderated neutron spectrum as can be found 
in nuclear fission reactors, with thermal $(<0.5 \mathrm{eV})$ and fast $(>1 \mathrm{MeV})$ fluence rates of $2.7 \times 10^{-8}$ and $4.6 \times 10^{-8} \mathrm{~cm}^{-}$ ${ }^{2} \mathrm{~S}^{-1}$, respectively. The contribution of the $(\alpha, n)$ from ${ }^{238} \mathrm{U}$ in the microphone is included in Fig. 6 in order to evidence the spreading of the source spectra features as neutrons are moderated in the hydrogenous gel.

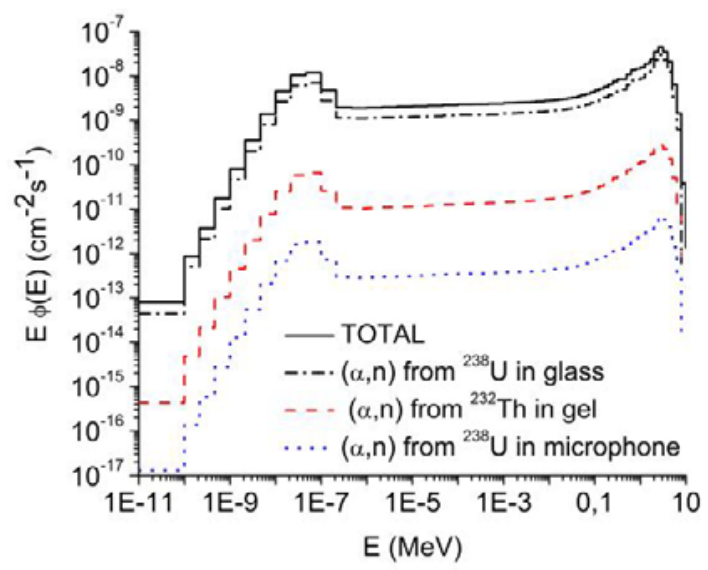

Fig. 6. Calculated on-detector neutron energy distribution, with discrimination of some contributing reactions.

The fluence rate beyond $43 \mathrm{keV}$ (the smallest $\mathrm{E}_{\min }$ ) is $7.0 \times 10^{-8} \mathrm{~cm}^{-2} \mathrm{~s}^{-1}$, of which $99 \%$ originate from the borosilicate glass container. The contribution of each reaction in borosilicate is the following: $7 \%$ from ${ }^{238} \mathrm{U}$ spontaneous fission, $62 \%$ from ${ }^{238} \mathrm{U}$ decay-induced $(\alpha, \mathrm{n})$ production and $31 \%$ from ${ }^{232} \mathrm{Th}$ decay-induced $(\alpha, n)$ production.

The energy distribution of recoils was extracted with MCNPX/PoliMI, with neutron and recoil energy cuts at 40 and $8 \mathrm{keV}$, respectively. Table 4 summarises the event rate per unit of liquid mass due to the various reactions and materials. The total event rate is $20 \%$ higher than previous calculations [20] mostly due to the application of increased $(\alpha, n)$ yields, thereby confirming the uncertainty estimate of $\sim 20 \%$ that was made then.

Table 4. Event rate (count $\mathrm{kg}^{-1} \mathrm{~d}^{-1}$ ) from spontaneous fission and decay-induced $(\alpha, n)$ reactions due to the presence of emitters in the SDD materials. Secular equilibrium is assumed for ${ }^{238} \mathrm{U}$ and ${ }^{232} \mathrm{Th}$ decay chains.

\begin{tabular}{|c|c|c|c|c|}
\hline Material & $\begin{array}{c}{ }^{238} \mathbf{U} \\
\text { sp.fiss. }\end{array}$ & $\begin{array}{c}{ }^{238} \mathbf{U} \\
(\boldsymbol{\alpha}, \mathbf{n})\end{array}$ & $\begin{array}{c}{ }^{232} \mathbf{T h} \\
(\boldsymbol{\alpha}, \mathbf{n})\end{array}$ & $\begin{array}{c}\mathbf{n a t}^{\mathbf{S m}} \\
(\boldsymbol{\alpha}, \mathbf{n})\end{array}$ \\
\hline Borosilicate & $\begin{array}{c}3.75 \\
\mathrm{x} 10^{-2}\end{array}$ & 0.288 & 0.144 & - \\
\hline Gel & $\begin{array}{c}<3.81 \\
\times 10^{-4}\end{array}$ & $\begin{array}{c}<5.53 \\
\times 10^{-4}\end{array}$ & $\begin{array}{c}<3.23 \\
\mathrm{x} 10^{-4}\end{array}$ & $\begin{array}{c}<3.74 \\
\times 10^{-8}\end{array}$ \\
\hline Microphone & $\begin{array}{c}<.91 \\
\mathrm{x} 10^{-6}\end{array}$ & $\begin{array}{c}<7.49 \\
\mathrm{x} 10^{-5}\end{array}$ & - & - \\
\hline TOTAL & \multicolumn{5}{|c|}{$0.41 \pm 20 \%$} \\
\hline
\end{tabular}

We thereby calculate a detector signal originating from its own emitters of $5.9 \times 10^{-3}$ counts per day for a 1 liter device with $14.3 \mathrm{~g}$ of $\mathrm{C}_{2} \mathrm{ClF}_{5}$ (corresponding to the average mass in the experiment). The calculated neutron detection efficiency, i.e., counts per neutron entering the gel volume) is $0.6 \%$. With a detector surface of $\sim 667$ $\mathrm{cm}^{2}$ a detection limit of $\sim 2 \times 10^{-8} \mathrm{n} \mathrm{cm}^{-2} \mathrm{~s}^{-1}$ is derived. This is two orders of magnitude lower than the neutron fluence rate in underground facilities at moderate (1-2 $\mathrm{km}$ water-equivalent) depth [20, 42]. In spite of the reduced detection efficiency, the time required for the characterization of such neutron environments $(\sim 1$ count/day) in 10 energy groups with 10 detectors operating in the varying temperature method is $\sim 2$ weeks, which is easily manageable with remote monitoring and control such as that employed e.g. in the SIMPLE experiments that run continuously over $\sim 3$ months.

Possibilities for future reduction of the background noise should focus on detector container materials at decreased emitter and Boron concentrations. Polymethylmethacrylate samples from various producers have been evaluated by neutron activation analysis yielding less than $0.05 \mathrm{ppm}{ }^{238} \mathrm{U}$. With a $(\alpha, \mathrm{n})$ yield $\sim 5 \%$ than that of borosilicate glass, an overall reduction by two orders of magnitude is in principle achievable - this corresponds to the upper limit for the signal induced by the detector gel itself.

\section{Conclusions}

The intrinsic neutron background signal of $\mathrm{C}_{2} \mathrm{ClF}_{5}$ SDDs was measured in an underground facility and simulated using the MCNPX/PoliMI code. The measured and calculated event rates were 0.15 and 0.41 count $\mathrm{kg}^{-1} \mathrm{~d}^{-1}$, respectively. This level of agreement is satisfactory for rare event measurements: should there be one more neutron detected and the agreement would be within the uncertainty of the calculation (dominated by that in $(\alpha, n)$ yields). The detection limit in the order of $10^{-8} \mathrm{~cm}^{-2} \mathrm{~s}^{-1}$ is is 2 orders of magnitude higher than the neutron environment in underground facilities at moderate depth. The device is therefore adequate to the characterisation of various facilities for low radiation background applications. If necessary, the current device can be upgraded for a significant background reduction simply by modifying the container material.

\section{Acknowledgements}

We thank Vitaly Kudryavtsev (University of Sheffield) for offering the improved version of SOURCES-4A and Gennadiy Vlaskin (ICTP, Moscow) for the interpretation of the $(\alpha, n)$ distributions. Luís Alves $\left(\mathrm{C}^{2} \mathrm{TN}\right)$ is acknowledged for the microscope usage.

This work was supported by Fundação para a Ciência e Tecnologia (Portuguese Ministry of Science) via grants IF/00628/2012/CP0171/CT0008 and SFRH/BPD/94028/ 2013 and projects UID/Multi/04349/2013 and PTDC/ EEI-ELC/2468/2014.

\section{References}

1. R.E. Apfel, Nucl. Instrum. Methods 162, 603 (1979) 
2. H. Ing, H.C. Birnboim, Nucl. Tracks Radiat. Meas. 8, 285 (1984)

3. F. d'Errico, Nucl. Instrum. Methods B184, 229 (2001)

4. H.W. Bonin, G.R. Desnoyers, T. Cousins, Radiat. Prot. Dosim. 46, 265 (2001)

5. F. d'Errico, W.G. Alberts, G. Curzio, S. Guldbakke, H. Kluge, M. Matzke, Radiat. Prot. Dosim. 61, 159 (1995)

6. F. d'Errico, A. Prokofiev, A. Sanniko, H. Schuhmacher, Nucl. Instrum. Methods A505, 50 (2003)

7. S.L. Guo, et al., Radiat. Meas. 31, 167 (1999)

8. S. Archambault, et al., Phys. Lett. B 711, 153 (2012)

9. M. Felizardo, et al., Phys. Rev. D 89, 072013 (2014)

10. A.C. Fernandes, et al., E3S Web of Conferences: iDUST 2016 (in press)

11. F. Seitz, Phys. Fluids 1, 2 (1958)

12. F.J. Maringer, D. Arnold, U. Wätjen, Appl. Radiat. Isot. 109 (2016)

13. A. Lesea, K. Castellani-Coulié, G. Waysand, J. Le Mauff, C. Sudre, IEEE Trans. Nucl. Sci. 55, 2148 (2008)

14. N. Fornengo, M. Regis, H.-S. Zechlin, J. Phys. Conf. Ser. 718 (2016)

15. http://lsbb.oca.eu

16. A.C. Fernandes, M. Felizardo, A. Kling, J.G. Marques, T. Morlat, Nucl. Instr. Meth. A 623, 960 (2010).

17. A.C. Fernandes, et al., E3S Web of Conferences 4, 03003 (2014)

18. T.A Girard, M. Felizardo, A.C. Fernandes, T.A. Morlat, A.R. Ramos, Proc. i-DUST 2010, 03003 (2011). http://dx.doi.org/10.1051/idust/201103003

19. X-5 Monte Carlo Team, LA-UR-03-1987, Los Alamos National Laboratory, 2003

20. A.C. Fernandes, et al., Astrop. Phys. 76, 43 (2016)

21. F.W. Stallmann, NUREG/CR-4349, ORNL/TM9333, Oak Ridge National Laboratory, 1985.

22. L.R. Greenwood, R.K. Smither, ANL/FPP/TM-197, Argonne National Laboratory, 1985

23. D.M. Mei, C.F. Zhang, A. Hime, Nucl. Instrum. Methods A 606, 651 (2009). Data available at http://neutronyield.usd.edu

24. M. Felizardo, et al., E3S Web of Conferences: iDUST 2016 (in press)

25. S.A. Pozzi, S.D. Clarke, W.J. Walsh, et al., Nucl. Instrum. Methods A 694, 119 (2012)

26. V. Tomasello, V.A. Kudryavtsev, M. Robinson, Nucl. Instrum. Methods A 595, 431 (2008)

27. T. Mayer-Kuckuk, Kernphysik (Teybner, Stuttgart, 1984)

28. M. Felizardo, et al., Nucl. Instrum. Methods A 585, $61(2008)$

29. T. Morlat, D. Limagne, G. Waysand, J.I. Collar, Nucl. Instrum. Meth. A 560, 339 (2006)

30. M. Felizardo, et al., Nucl. Instrum. Methods A 589, 72 (2008)

31. M. Felizardo et al., Phys. Rev. Lett. 105, 211301 (2010)

32. T.A. Girard, et al., Phys. Rev. Lett. 108, 259002 (2012)
33. M. Felizardo, et al., Astrop. Phys. (to be published)

34. A.R. Ramos et al., Radiat. Prot. Dosim. 115, 398 (2005)

35. V.F. Weigel, Chem. Ztg. 102, 287 (1978)

36. D.M. Mei, A. Hime, Phys. Rev. D 83, 053004 (2006)

37. Y.F. Wang, V. Balic, G. Gratta, A. Fassó, S. Roesler, A. Ferrari, Phys Rev D 64, 013012 (2001)

38. L.S. Waters, G.W. McKinney, J.W. Durkee, M.L. Fensin, J.S. Hendricks, M.R. James, R.C. Johns, D.B. Pelowitz, AIP Conf. Proc. 896, 81 (2007)

39. P. Jagam, J.J. Simpson, Nucl. Instrum Methods A 324, 389 (1993). Erratum: Nucl. Instrum. Methods A 334, 657 (1993)

40. A.C. Fernandes, A. Kling, G.N. Vlaskin (this volume)

41. G.N. Vlaskin, Yu.S. Khomyakov, V.I. Bulanenko, Atomic Energy 117, 357 (2015)

42. S. Cebrián, B. Beltrán, J. M. Carmona, et al., Nuclear Physics B (Proc. Suppl.) 138 (2005) 65-67 